OPEN ACCESS

Edited by: Sigmar Stricker,

Freie Universität Berlin, Germany

Reviewed by:

Gillbert Weidinger,

University of UIm, Germany

Rebecca Richardson,

University of Bristol, United Kingdom

*Correspondence:

José Luis de la Pompa

jlpompa@cnic.es

Specialty section:

This article was submitted to

Signaling,

a section of the journal

Frontiers in Cell and Developmental

Biology

Received: 18 February 2021

Accepted: 07 April 2021

Published: 07 May 2021

Citation:

Grivas D, González-Rajal Á and

de la Pompa JL (2021) Midkine-a Regulates the Formation of a Fibrotic

Scar During Zebrafish Heart

Regeneration.

Front. Cell Dev. Biol. 9:669439.

doi: 10.3389/fcell.2021.669439

\section{Midkine-a Regulates the Formation of a Fibrotic Scar During Zebrafish Heart Regeneration}

\author{
Dimitrios Grivas ${ }^{1,2,3}$, Álvaro González-Rajal1,4,5 and José Luis de la Pompa ${ }^{1,2 *}$ \\ 1 Intercellular Signalling in Cardiovascular Development and Disease Laboratory, Centro Nacional de Investigaciones \\ Cardiovasculares Carlos III (CNIC), Madrid, Spain, ${ }^{2}$ Ciber de Enfermedades Cardiovasculares, Madrid, Spain, \\ ${ }^{3}$ Developmental Biology, Clinical, Experimental Surgery and Translational Research Center, Biomedical Research Foundation \\ Academy of Athens, Athens, Greece, ${ }^{4}$ Genomics and Epigenetics Division, Garvan Institute of Medical Research, Sydney, \\ NSW, Australia, ${ }^{5}$ St Vincent's Clinical School, Faculty of Medicine, University of New South Wales, Sydney, NSW, Australia
}

Unlike the hearts of mammals, the adult zebrafish heart regenerates after injury. Heart cryoinjury in zebrafish triggers the formation of a fibrotic scar that gradually degrades, leading to regeneration. Midkine-a (Mdka) is a multifunctional cytokine that is activated after cardiac injury. Here, we investigated the role of $m d k a$ in zebrafish heart regeneration. We show that mdka expression was induced at 1-day post-cryoinjury (dpci) throughout the epicardial layer, whereas by $7 \mathrm{dpci}$ expression had become restricted to the epicardial cells covering the injured area. To study the role of mdka in heart regeneration, we generated maka-knock out $(\mathrm{KO})$ zebrafish strains. Analysis of injured hearts showed that loss of mdka decreased endothelial cell proliferation and resulted in an arrest in heart regeneration characterized by retention of a collagenous scar. Transcriptional analysis revealed increases in collagen transcription and intense TGF $\beta$ signaling activity. These results reveal a critical role for mdka in fibrosis regulation during heart regeneration.

Keywords: zebrafish heart regeneration, fibrotic scar, Midkine-a, signaling/signaling pathways, epicardium, collagen

\section{INTRODUCTION}

The adult mammalian heart has limited regeneration capacity, and myocardial infarction (MI) generates a permanent fibrotic scar that progressively leads to heart failure (Benjamin et al., 2019). Conversely, zebrafish can regenerate damaged cardiac tissue (Poss et al., 2002). Cryoinjury to the zebrafish ventricle results in massive cell death and the formation of scar tissue that gradually resolves, resulting in complete heart regeneration within 90 days (Schnabel et al., 2011; Chablais and Jazwinska, 2012b; Gonzalez-Rosa and Mercader, 2012). The most abundant extracellular matrix (ECM) component responsible for fibrotic tissue formation is collagen (Theocharis et al., 2016). Fibrosis progression is also dependent on the ECM molecule periostin, which promotes collagen cross-linking and fibroblast activation (Ito et al., 2014; Sanchez-Iranzo et al., 2018). In the injured zebrafish heart, the main sources of ECM are epicardial cells and epicardial-derived fibroblasts (Wang et al., 2013; Marro et al., 2016; Sanchez-Iranzo et al., 2018). TGFß signaling, important for matricellular protein production, is active during regeneration, and chemical inhibition of the 
pathway decreases collagen deposition and blocks regeneration (Chablais and Jazwinska, 2012b). Heart regeneration is also prevented by inhibition of fibrotic tissue resolution ( $\mathrm{Xu}$ et al., 2018), indicating that heart regeneration requires both the formation and the removal of the scar tissue.

Midkine $(m d k)$ is a small, pleiotropic cytokine that is upregulated after injury in several tissues, including the heart, fin and retina (Lien et al., 2006; Luo et al., 2012). Cardiac insult in Midkine-KO mice leads to larger injuries and decreased heart function (Horiba et al., 2006; Ishiguro et al., 2011). Prolonged exposure of injured hearts to Midkine results in reduced collagen deposition in both the infarcted and healthy areas of the ventricle (Fukui et al., 2008; Takenaka et al., 2009; Sumida et al., 2010). Additionally, Midkine improves angiogenesis in injured cardiac tissue (Fukui et al., 2008; Takenaka et al., 2009; Sumida et al., 2010), indicating the importance of Midkine in the cardiac response to injury in mammals. Zebrafish have two Midkine genes, midkine-a (mdka) and its paralog midkine-b (mdkb), which during early development show complementary patterns of expression in the neural tube (Winkler et al., 2003). In the absence of $m d k a$, retina regeneration is impaired, evidenced by decreased Muller glia proliferation and impaired photoreceptor regeneration (Nagashima et al., 2020). Fin regeneration is also delayed, due to an initial decrease in cell proliferation, although ultimately the fin is fully regenerated (Ang et al., 2020). Despite its importance during the cardiac response to injury in mammals, little is known about the role of $m d k a$ in zebrafish heart regeneration.

Here, we generated maka-KO zebrafish and studied heart regeneration. We found that $m d k a$ is upregulated upon injury in the activated epicardium. Deletion of $m d k a$ decreased endothelial cell proliferation and prevented heart regeneration, leading to a persistent injured area enriched in collagen deposition. Transcriptional analysis revealed intense TGF $\beta$ signaling activity and increased expression of ECM molecules, including collagen and periostin, revealing a crucial role for $m d k a$ in the regulation of the fibrotic response upon heart injury.

\section{RESULTS}

\section{mdka Expression in the Regenerating Heart}

The expression of $m d k a$ has been reported to be upregulated upon heart injury in several animal models (Horiba et al., 2006; Lien et al., 2006; Fukui et al., 2008; Sumida et al., 2010). We therefore performed a quantitative PCR (qPCR) analysis in cryoinjured zebrafish hearts to analyse $m d k a$ expression during regeneration (Figure 1A). $m d k a$ RNA was already expressed in the heart at 1-day post-cryoinjury (dpci), reaching a peak at $3 \mathrm{dpci}$, followed by a slight reduction at $7 \mathrm{dpci}$. We next investigated the expression pattern of $m d k a$ by in situ hybridization (ISH) (Figures 1B-D'). At $1 \mathrm{dpci}$, $m d k a$ expression was detected in the epicardium, with no expression in intact hearts (Supplementary Figures $\mathbf{1} \mathbf{A}, \mathbf{A}^{\prime}$ and Figures $\mathbf{1 B}, \mathbf{B}^{\prime}$ ). At $3 \mathrm{dpci}, m d k a$ expression was activated in the epicardium, and by $7 \mathrm{dpci}$ the expression became restricted to the multi-layered epicardium covering the injury site (Figures $\mathbf{1 C}-\mathbf{D}^{\prime}$ ). Epicardial cells maintained $m d k a$ expression at $14 \mathrm{dpci}$ (Supplementary Figures $\mathbf{1 B}, \mathbf{B}^{\prime}$ ), and $m d k a$ remained detectable up to 130 dpci in the epicardium, the area between the cortical and trabecular myocardium, and in the compact layer, hinting at vascular expression (Supplementary Figures $\mathbf{1 C}, \mathbf{C}^{\prime}$ ). To further define the epicardial localization of $m d k a$, we performed fluorescence ISH in heart sections of $\mathrm{Tg}(w t 1 b$ :GFP) zebrafish, which express GFP in $w t 1 b^{+}$epicardial cells after injury (Gonzalez-Rosa et al., 2011). mdka ISH was followed by immunostaining for GFP and Aldh1a2, an enzyme involved in retinoid acid synthesis that is expressed in epicardial cells after injury (Kikuchi et al., 2011; Figures 1E-F' ${ }^{\prime \prime}$ ). mdka was expressed in $\mathrm{GFP}^{+}$cells (Figures $\mathbf{1 F}-\mathbf{F}^{\prime \prime}$, arrowheads) and in Aldh1a2 expressing cells (Figures $\mathbf{1 F}^{\prime}, \mathbf{F}^{\prime \prime}$, arrows), indicating that injury triggers $m d k a$ expression in signaling cells. Our results thus show that $m d k a$ is highly upregulated in the epicardium upon injury and later persists in the activated epicardium.

\section{Generation of $\boldsymbol{m d k a}$ Knock-Out Zebrafish}

In zebrafish, $m d k a$ is not expressed in the embryonic (Winkler et al., 2003) or adult heart; however, our initial analysis showed that $m d k a$ expression is readily activated in the epicardium after heart injury (Figure 1). The specific induction of high mdka expression upon injury suggested that loss of $m d k a$ might have a significant effect on heart regeneration. Therefore, we generated mdka knock-out (KO) zebrafish using CRISPR/Cas9 technology (Figure 2). The $m d k a$ gene is composed of five exons, and we targeted the third, which corresponds to the N-terminus of the protein (Figure 2A). Gene editing efficiency was high and resulted in equal numbers of deletion and insertion mutations (Figure 2A). Most of the predicted mdka mutations had a premature stop codon due to a frame shift in the open reading frame (Figure 2B). We selected the mutant allele $m d k a^{c n 105}$ for further characterization (Figures 2A,B). This mutant allele results in severely decreased $m d k a$ expression, as revealed by whole-mount ISH (WM-ISH) and qPCR analysis (Figures 2CE). Loss of $m d k a$ did not affect embryonic development, and $m d k a^{c n 105}$ mutants were viable and fertile. We next used qPCR and WM-ISH to examine whether the absence of $m d k a$ triggered upregulation of $m d k b$. qPCR revealed no change in $m d k b$ expression, and WM-ISH confirmed similar $m d k b$ expression in $m d k a^{+/+}$fish and $m d k a^{c n 105}$ mutants (Figure 2E and Supplementary Figures 2A,B). To confirm that Mdka protein expression was lost, we conducted an immunofluorescence analysis of cryoinjured hearts from $m d k a^{+/+}$and $m d k a^{c n 105}$ fish (Figures 2F,G). The strong epicardial expression of Mdka at 7 dpci in $m d k a^{+/+}$hearts was completely absent in their $m d k a^{c n} 105$ counterparts. Loss of Mdka was confirmed by western blot (WB) analysis (Figure $2 \mathbf{H}$ ), which showed decreased post-injury Mdka expression in $m d k a^{+/ c n 105}$ hearts and no expression in $m d k a^{c n 105}$ hearts. Furthermore, expression of $m d k b$ in $m d k a^{c n 105}$ injured hearts was similar to that in $m d k a^{+/+}$hearts (Supplementary Figures 2C,D), indicating that the $m d k a^{c n 105}$ mutation abolishes 

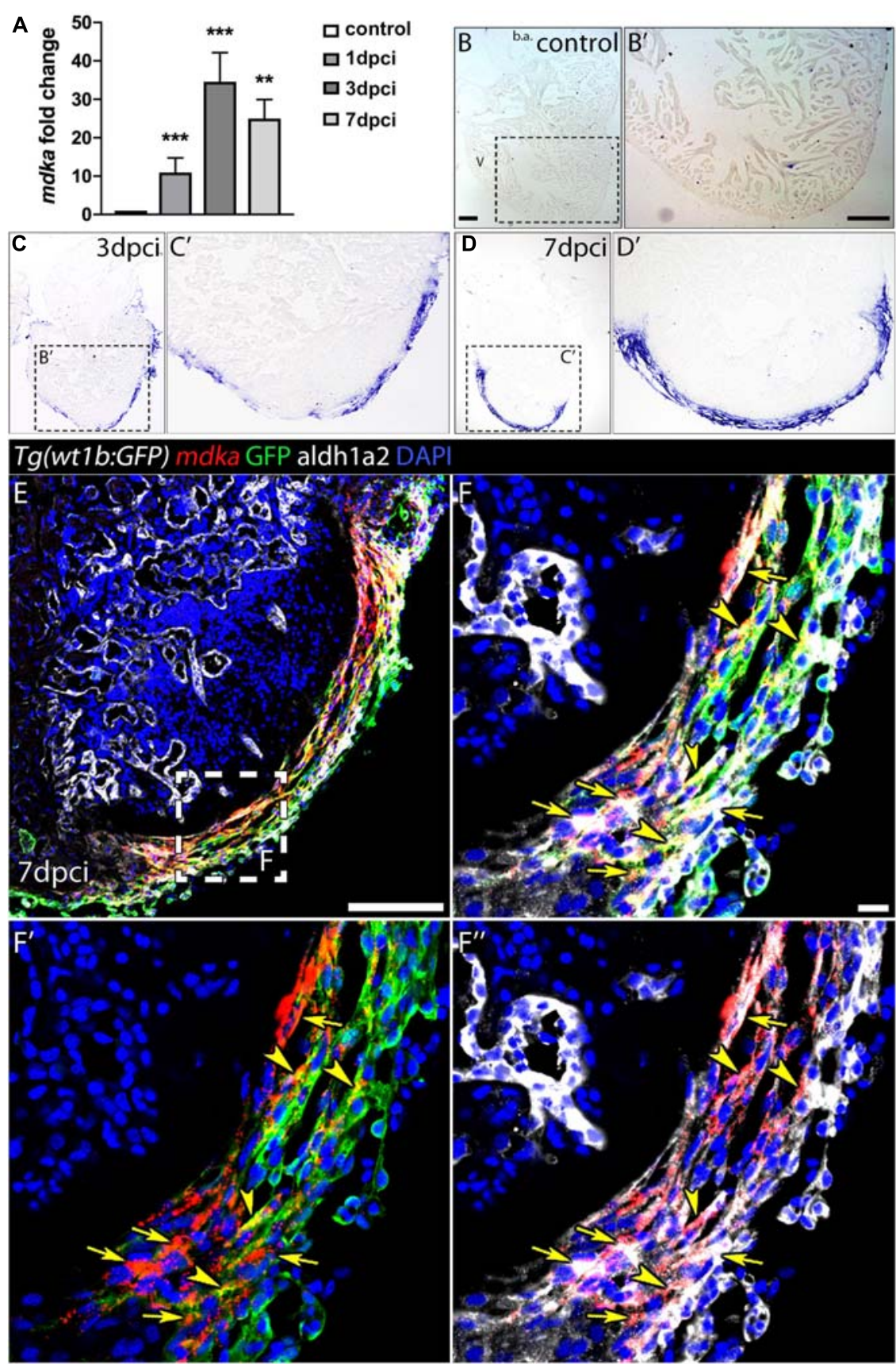

FIGURE 1 | $m$ dka expression upon injury. (A) qPCR analysis of $m d k a$ in regenerating hearts. Brown-Forsythe and Welch ANOVA tests; ${ }^{\star \star} P<0.01$, ${ }^{\star \star \star} P<0.001$;

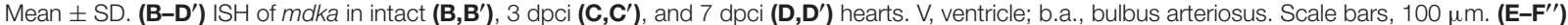

Fluorescent ISH for mdka followed by immunolabeling for GFP and Aldh1a2 in 7 dpci Tg(wt1b:GFP) hearts. Arrowheads indicate mdka-GFP overlap and arrows mdka-Aldh1a2 overlap. Scale bar E, $100 \mu \mathrm{m}$; (F-F'), $10 \mu \mathrm{m}$.

$m d k a$ transcript and protein expression without upregulating potentially compensatory $m d k b$ expression.

\section{mdka-KO Hearts Fail to Regenerate}

We next examined the effect of mdka inactivation on heart regeneration. Hearts from $m d k a^{+/+}, m d k a^{c n 105}$, and $m d k a^{+/ c n 105}$ animals were cryoinjured and allowed to regenerate for 90-days. Hearts were harvested and processed for Acid Fuchsin Orange-G (AFOG) staining, which labels collagen, fibrin, and healthy tissue (Figures $\mathbf{3 A}-\mathrm{C}$ and Supplementary Figure 3 ). The analysis revealed that $m d k a^{c n 105}$ hearts had significantly larger scar area than their $m d k a^{+/+}$and $m d k a^{c n 105 /+}$ counterparts (Figure 3D). Additionally, the scar area of $m d k a^{c n 105}$ hearts was characterized by persistent 
A mdka

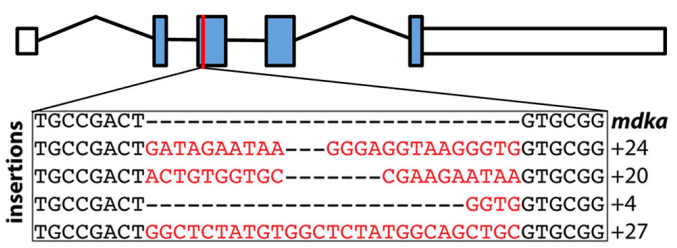

$\overline{\bar{\Xi}}$ AAAGAGAAGAATAAGGGAGGTAAGGGTGGTGCCGACTGTGCGGAGTGGCTCTATGGCAGCTGCG $\boldsymbol{m} \boldsymbol{d} \boldsymbol{k} \boldsymbol{a}$

AAAGAGAAGAATAAGGGAG-------------TGGAGCGGAGTGGCTCTATGGCAGCTGCG $-16 /+4$ cn 105

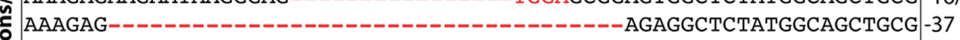

AAAGAGAAGAATAAGGGAGGTAAGGGTGGTGCCGACT----------------------GCTGCG -21

AAAGAGAAGAATAAGGGAGGTAAGGGTG---------GTGCGGAGTGGCTCTATGGCAGCTGCG -9

B

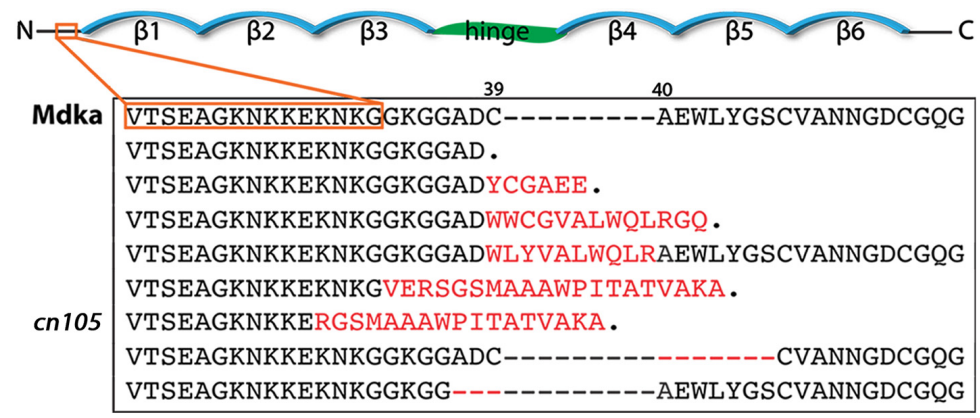

$\underline{m d k a}$

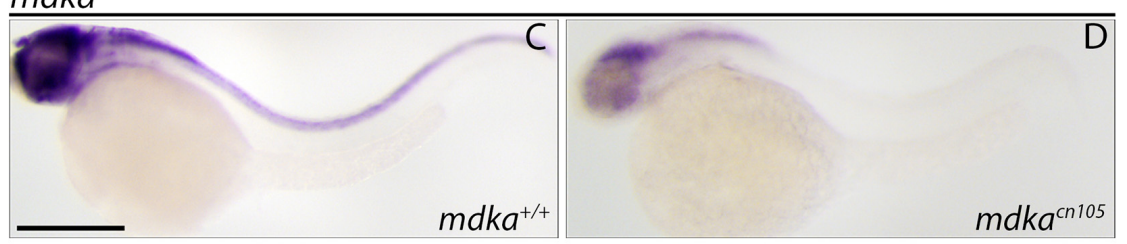

E

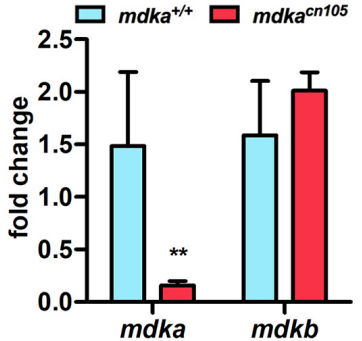

H

$7 \mathrm{dpci}$
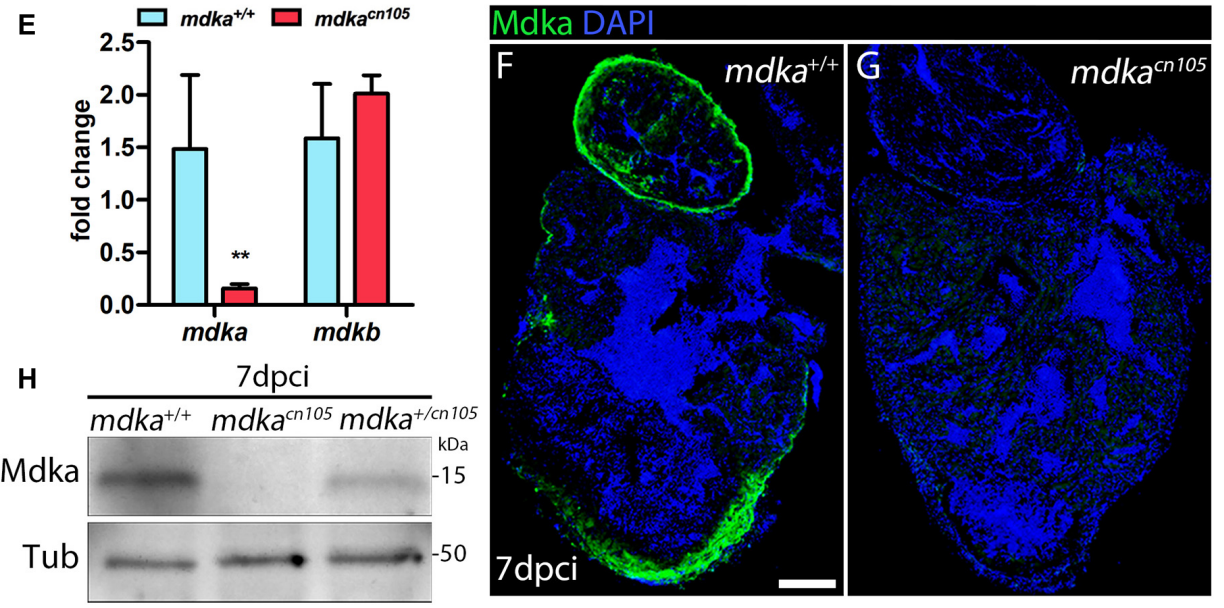

FIGURE 2 | Generation of $m d k a-K O$ zebrafish. (A) The $m$ dka locus and DNA mutations introduced by CRISPR/Cas9. The red line indicates the CRISPER target site, red letters indicate insertions, and red hyphens deletions. (B) Mdka domain organization and predicted protein mutations. Red letters denote novel amino acids, red hyphen deletions. $\beta$ stands for beta-strands and the hinge domain is shown in green. (C,D) Whole-mount ISH (WM-ISH) for mdka in 2-day post-fertilization (dpf) mdka ${ }^{+/+}$and $m d k a^{c n 105}$ embryos. Scale bar, $200 \mu \mathrm{m}$. (E) qPCR analysis of $m d k a$ and $m d k b$ in 2 dpf $m d k a^{+/+}$and $m d k a^{c n 105}$ embryos. $t$-test; ${ }^{* \star} P<0.01$; Mean \pm SD. (F,G) Immunofluorescence staining of Mdka in 7 dpci mdka ${ }^{+/+}$and mdka ${ }^{c n 105}$ hearts. Scale bar, $100 \mu \mathrm{m}$. (H) Western blot analysis for Mdka in 7 dpci $m d k a^{+/+}, m_{k} a^{c n 105}$, and $m d k a^{+/ c n 105}$ hearts. Tub, a-Tubulin; kDa, kilodalton.

collagen deposition compared with $m d k a^{+/+}$and $m d k a^{c n 105 /+}$ hearts (Figure 3E). These observations indicate that mdka deletion blocks heart regeneration. We further examined the regeneration of the caudal fin after amputation (Supplementary
Figure 4). Loss of $m d k a$ led to a delay in fin regeneration, with fin outgrowth significantly smaller at 7 days post-amputation (dpa); however, regeneration was completed by $14 \mathrm{dpa}$ (Supplementary Figure 4G), as reported previously (Ang et al., 2020). 


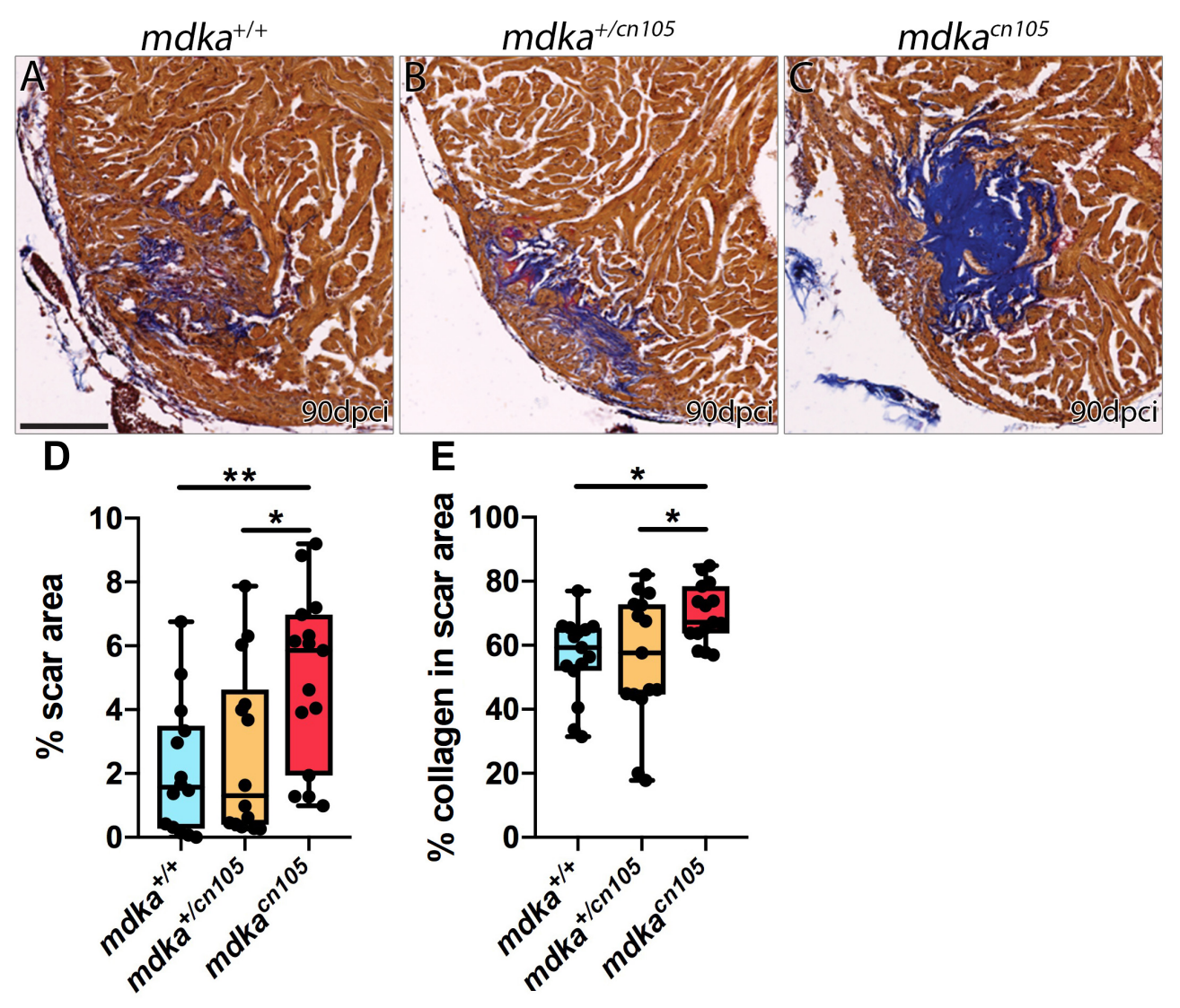

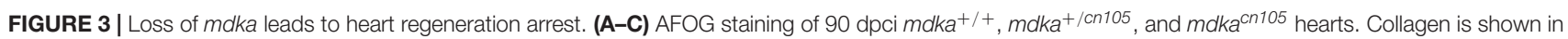
blue, fibrin in red, and healthy myocardium in brown. Scale bar, $100 \mu \mathrm{m}$. (D) Quantification of the scar area normalized to the total ventricle area. $n_{\mathrm{WT}}=14$, $n_{\mathrm{KO}}=15, n_{\mathrm{HET}}=14$. (E) Percentage of collagen in the scar area. $n_{\mathrm{WT}}=n_{\mathrm{KO}}=n_{\mathrm{HET}}=15$. Ordinary one-way anova; ${ }^{\star} P<0.05 ;{ }^{\star \star} P<0.01 ; \mathrm{Mean} \pm \mathrm{SD}$.

\section{Increased Fibrosis in Injured $m \mathbf{m a g}^{\mathrm{cn} 105}$ Hearts}

To further study the effect of $m d k a$ deletion, we compared the transcriptional profile of $7 \mathrm{dpci} m d k a^{+/+}$and $m d k a^{c n 105}$ ventricles by RNA-seq. The analysis identified 2,029 differentially expressed genes, 990 of them upregulated and 1,039 downregulated (Figure 4A and Supplementary Table 1). Ingenuity pathway analysis (IPA) identified enrichment of processes involved in heart failure, including cardiac fibrosis, heart degeneration, and vascular lesions (Figure 4B). Analysis of upstream regulators suggested increased activation of the TGF $\beta$ pathway, including Tgf $\beta 1$, Tgfbr1, and Smad downstream effectors such as Smad3 and Smad4, whereas the TGF $\beta$ inhibitory effector Smad7 was reduced (Figure 4C; Moustakas and Heldin, 2009).

In $m d k a^{c n 105}$ hearts, genes associated with fibrosis were significantly upregulated (Supplementary Figure 5). These included genes for the ECM components colla1a, colla1b, colla2, col5a1, col5a2a, and postna (Chablais and Jazwinska, 2012b; Ito et al., 2014). Also upregulated were pcolce $2 b$, cthrc1a and plod2, which are involved in collagen stability and scar tissue formation (Gilkes et al., 2013; Sorci-Thomas et al., 2015; Li et al., 2019). In contrast, expression of vegfaa and hiflaa, genes required for vascular development and repair, was decreased in $m d k a^{c n 105}$ hearts (Marin-Juez et al., 2016; Gerri et al., 2017; Marin-Juez et al., 2019).

We validated our RNA-seq results by qPCR analysis using a different set of samples that also showed increased expression of postnb, fibronectin1a and fibronectin1b (Figure 4D). We confirmed the transcriptional upregulation of several collagens, including the fibrillar collagens colla1a, colla1b, colla2, col5a1, and col5a $2 a$, which are found in type I fibrils (Figure 4D; Theocharis et al., 2016). In addition, AFOG staining of 7 dpci hearts showed increased collagen deposition in $m d k a^{c n 105}$ injured hearts (Supplementary Figure 6). The matricellular molecules postna and postnb were also upregulated in $m d k a^{c n 105}$ hearts (Figure 4D). Periostin, which is expressed by cardiac fibroblasts is involved in formation of fibrillar collagen and contributes to fibroblast stimulation and persistency in injured hearts (Shimazaki et al., 2008; Sanchez-Iranzo et al., 2018; Dixon et al., 2019). The secretory function of activated fibroblasts, which secrete the ECM proteins that form fibrotic tissue, depends on TGF $\beta$. Therefore, we evaluated TGF $\beta$ pathway activation by examining the downstream effector phospho-Smad3 (psmad3) (Supplementary Figure 7). Interestingly, the analysis revealed 

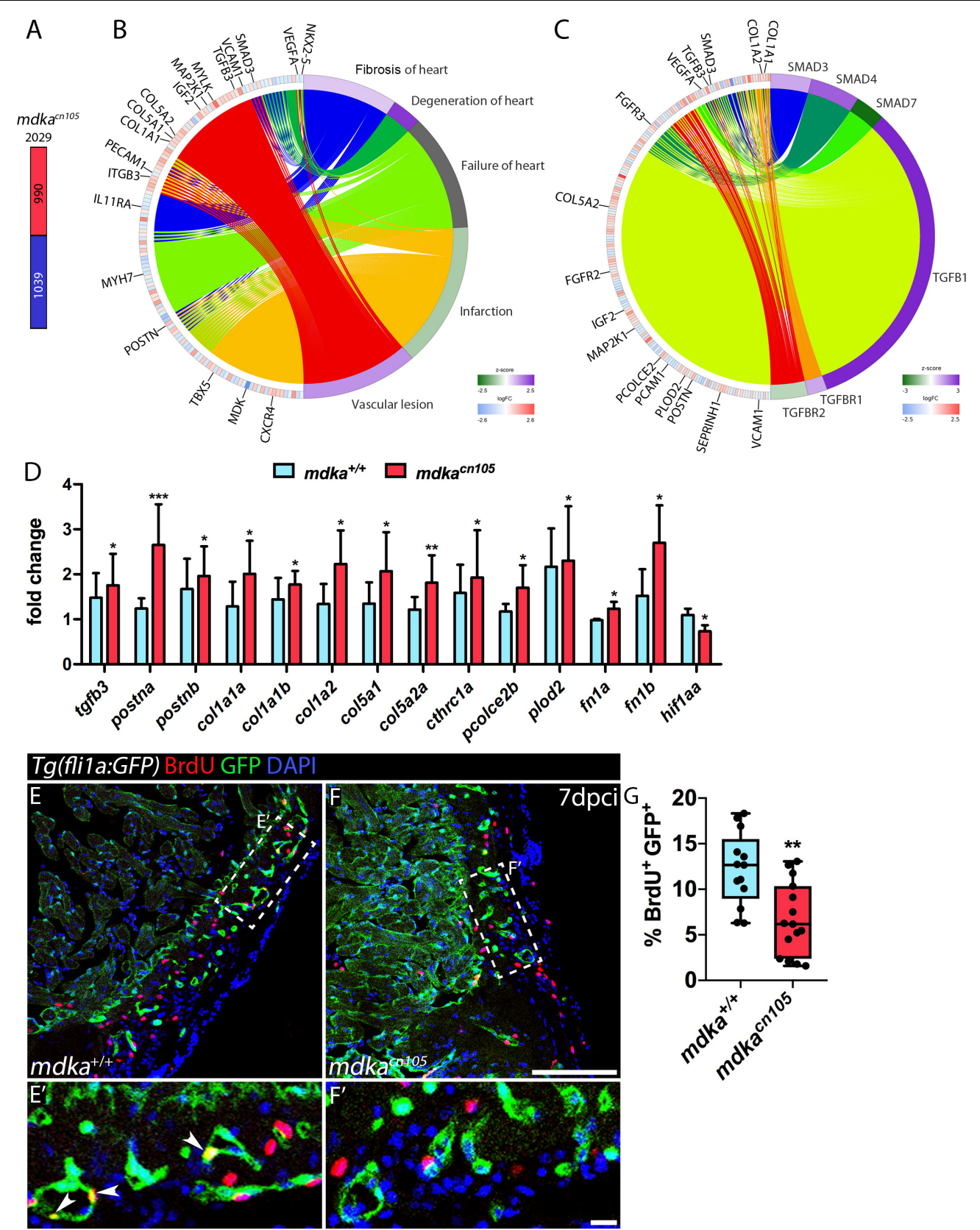

FIGURE 4 | Transcriptional profiling of regenerating $m d k^{c n 105}$ hearts. (A) Total number of differentially expressed genes identified by RNA-seq. Numbers indicate upregulated genes (red) and downregulated genes (blue). (B,C) Circular plots showing representative differentially expressed genes (left semicircle perimeter) and IPA biofunctions and upstream regulators (right semicircle perimeter). Activation z-score scale: green, repression; magenta, activation; white, unchanged. LogFC scale: red, upregulated; blue, downregulated; white, unchanged. (D) qPCR analysis of mdka ${ }^{+/+}$and $m d k a^{c n 105} 7$ dpci hearts. $t$-test; ${ }^{*} P<0.05,{ }^{* *} P<0.01$, ${ }^{* * *} P<0.001$; Mean \pm SD. (E,F) Immunofluorescence staining of GFP and BrdU in 7 dpci Tg(fli1a:GFP) heart sections. ( $\left.\mathbf{E}^{\prime}, \mathbf{F}^{\prime}\right)$ Higher magnification of the dashed lines in (A,F). Arrowheads indicate proliferating endothelial cells. Scale bar (E,F) $100 \mu \mathrm{m} ;\left(\mathbf{E}^{\prime}, \mathbf{F}^{\prime}\right) 10 \mu \mathrm{m}$. (G) Quantification of BrdU ${ }^{+} / \mathrm{GFP}^{+}$endothelial cells. $n_{\mathrm{WT}}=13, n_{\mathrm{KO}}=15$; $t$-test; ${ }^{* *} P<0.01 ;$ Mean \pm SD.

that phosphorylation of Smad3 was similar in $m d k a^{+/+}$and $m d k a^{c n} 105$ hearts, suggesting that $m d k a$ affects TGF $\beta$ activation through non-canonical pathways.

We next examined the proliferation of epicardial cells, because of their expression of $m d k a$, and cardiomyocytes; however, neither cell type was significantly affected in mdka mutants (Supplementary Figure 7), suggesting that Mdka from epicardial or epicardial-derived cells is not required for the initial proliferation burst in the epicardium or in cardiomyocytes. Since Midkine is known to act as a pro-angiogenic factor 
(Sumi et al., 2002), we also analyzed the proliferation of cortical endothelial cells. We found that endothelial cells adjacent to the injury site proliferate less in $m d k a^{c n 105}$ hearts than in control hearts (Figures $4 \mathrm{E}-\mathbf{G}$ ). In addition, we detected reduced transcriptional expression of hiflaa in $m d k a^{c n 105}$ hearts (Figure 4D). HIF-1a has been shown to positively regulate Midkine expression and leads to enhanced angiogenesis (Reynolds et al., 2004), and zebrafish hifla mutants have reduced endothelial cell proliferation in regenerating hearts (Marin-Juez et al., 2019). The decreased expression of hiflaa in $m d k a^{c n 105}$ regenerating hearts can thus provide an explanation for the decline in endothelial cell proliferation.

Transcriptional analysis of injured $m d k a^{c n 105}$ hearts revealed that loss of $m d k a$ led to upregulation of ECM moleculesincluding collagens, periostins and fibronectins-and intense TGF $\beta$ pathway activity. Additionally, the proliferation of the cortical endothelial cells was decreased, suggesting defective revascularization in the regenerating hearts. Therefore, increased fibrosis and persistent scar in $m \mathrm{mka}^{c n 105}$ mutant hearts results from contributions of both increased ECM expression and angiogenesis defects.

\section{DISCUSSION}

Midkine is a highly conserved secreted factor that is induced upon injury of different tissues including muscle, retina, fin, and heart (Winkler et al., 2003; Lien et al., 2006; Luo et al., 2012; Ikutomo et al., 2014; Ang et al., 2020). Here, we generated $m d k a-K O$ zebrafish to study the role of Mdka in heart regeneration upon cryoinjury. Deletion of $m d k a$ resulted in arrest of heart regeneration and a scar area with increased collagen deposition. Analysis of mdka-deficient hearts showed transcriptional upregulation of ECM components such as collagens, periostins and fibronectins. Additionally, the proliferation of cortical endothelial cells was decreased, indicating defective angiogenesis.

Expression analysis revealed that $m d k a$ is not expressed in intact adult heart but it is highly upregulated upon injury. $m d k a$ expression was detected at $1 \mathrm{dpci}$ throughout the epicardium, was maintained at $3 \mathrm{dpci}$, and by $7 \mathrm{dpci}$, the expression was restricted to epicardial cells surrounding the damaged tissue. Additionally, after the end of regeneration at $130 \mathrm{dpci}$, we also detected $m d k a$ in the newly formed compact layer, possibly in the vasculature, as well as in the zone between the cortical and trabecular myocardium, suggesting that regeneration requires prolonged $m d k a$ expression. This distinct spatio-temporal expression pattern contrasts with data from injured rodent hearts, which show $M d k$ expression in cardiomyocytes and/or endothelial cells, but not in epicardial cells (Obama et al., 1998; Fukui et al., 2008). In 7 dpci zebrafish hearts, the epicardium consists of a heterogeneous cell population that includes macrophages and fibroblasts (Sanchez-Iranzo et al., 2018; Sanz-Morejon et al., 2019). The main source of ECM is fibroblasts (Kendall and Feghali-Bostwick, 2014), and RNA-seq data from postn $b^{+}$cells show that $m d k a$ is highly upregulated in epicardial-derived fibroblasts in regenerating zebrafish hearts (Sanchez-Iranzo et al., 2018). $m d k a$ is also widely expressed in the epicardium, and epicardial cells secrete ECM components (Wang et al., 2013), suggesting that $m d k a$ is involved in scar tissue formation by epicardial and epicardial-derived cells.

To study the function of $m d k a$ in heart regeneration, we generated $m d k a-K O$ zebrafish using CRISPR/Cas9 genetic editing. $m d k a^{c n 105}$ embryos developed and reached adulthood. Loss of mdka did not affect the transcription of the paralog $m d k b$ in embryos. Mdka and Mdkb share 68\% amino acid identity, and they show non-overlapping expression patterns in the embryos (Winkler et al., 2003). In the adult heart, we detected $m d k a$ expression only after cardiac injury, suggesting that $m d k a$ deletion will adversely affect heart regeneration. In $m d k a^{c n 105}$ zebrafish, Mdka protein expression was lost in the injured heart without any accompanying activation of $m d k b$, suggesting that functional redundancy in the heart from $m d k b$ is unlikely.

Cryoinjury of $m d k a^{c n 105}$ hearts resulted in incomplete regeneration at $90 \mathrm{dpci}$, the final time-point of the regeneration process after cryoinjury. The persistent scar was characterized by the presence of collagen. Gene expression analysis showed that ECM molecules including colla1a, colla1b, colla2, col5a1, col5a2a, postna, postnb, fibronectin1a, and fibronectin1b were upregulated. TGF $\beta$ induces transcription of collagens type I and V (Verrecchia et al., 2001). Upon heart cryoinjury in zebrafish, $\operatorname{tg} f \beta 1$ is expressed in the injured area, and inhibition of TGF $\beta$ signaling leads to decreased collagen deposition and impairs regeneration (Chablais and Jazwinska, 2012b). Intriguingly, analysis of the TGF $\beta$ downstream effector Smad3 revealed comparable phosphorylation of Smad3 in $m d k a^{c n 105}$ and $m d k a^{+/+}$hearts, suggesting that the effect on the TGF $\beta$ pathway is via non-canonical pathways. Collagen is the main ECM scaffolding protein, and our analysis shows that collagen is upregulated in injured $m d k a^{c n 105}$ hearts. Specifically, fibril-forming collala and collalb encode procollagen chains that combine with the colla2 chain to produce type I procollagen molecules. Through enzymatic process, these procollagen molecules produce strong crosslinked fibers that contribute to scarring (Holmes et al., 2018), and these molecules are upregulated after mdka deletion. $m d k a^{c n 105}$ hearts also showed upregulation of postna and postnb. In injured hearts, periostin is expressed by epicardialderived fibroblasts and is involved in fibroblasts activation and persistence during regeneration (Sanchez-Iranzo et al., 2018; Dixon et al., 2019). Periostin is also involved in fibrosis through its stimulation of fibrillar collagen formation (Shimazaki et al., 2008). Also upregulated were pcolce2b, cthrcla, and plod2. pcolce $2 b$ is involved in collagen-fibril assembly and ECM formation, is expressed in the epicardium after heart injury in zebrafish, and has been linked to $\operatorname{Tgf} \beta 1$ stimulation and to pancreatic fibrotic disease (Cao et al., 2016). cthrcla has been shown to contribute to fibrosis progression via TGF $\beta$ dependent enhancement of liver fibrosis (Li et al., 2019), and plod2 is involved in collagen fiber architecture and fibrosis development (Gilkes et al., 2013). These data indicate that 
$m d k a$ deletion triggers upregulation of genes responsible for scar formation and stability.

In zebrafish, heart regeneration after injury depends on both the formation and degradation of the scar tissue (Chablais and Jazwinska, 2012a; Sanchez-Iranzo et al., 2018; Xu et al., 2018). We found that mdka expression overlaps with the epicardial markers Wt1b and Aldh1a2, which are also coexpressed with $t c f 21$; moreover, $t c f 21^{+}$epicardial cells participate in collagen deposition (Kikuchi et al., 2011; Wang et al., 2013; Sanchez-Iranzo et al., 2018). Moreover, transcriptome analysis of post $1 b^{+}$fibroblasts revealed that $m d k a$ is highly expressed by these cells (Sanchez-Iranzo et al., 2018). This suggests that heart regeneration arrest in $m d k a$-deficient zebrafish is due to an increased fibrotic response, as evidenced by upregulation of collagens and periostin. This is consistent with findings in the injured mammalian heart, where treatment with Midkine after MI leads to decreased collagen deposition (Takenaka et al., 2009; Sumida et al., 2010). Additionally, postn $^{+}$fibroblasts are detectable until $90 \mathrm{dpci}$, which could explain the persistent $m d k a$ expression that we observed after regeneration was complete. These data suggest that ECM regulation requires $m d k a$ expression by epicardial cells and epicardial-derived fibroblasts throughout the course of regeneration, and that $m d k a$ loss leads to increased ECM protein expression, resulting in impaired regeneration. An interesting idea is that $m d k a$ might be also involved in scar removal, and $m d k a$ inactivation result in a persistent collagen-rich scar. In this regard, treatment of human skin fibroblasts with Midkine results in increased MMP2 transcription (Yamada et al., 1997). Future experiments will shed light on this issue.

We also found that $m d k a^{c n 105}$ hearts have reduced transcriptional expression of the angiogenesis promoter hiflaa. Angiogenesis is necessary for revascularization, and defects in this process results in arrest of regeneration and maintenance of the fibrotic tissue (Harrison et al., 2015; MarinJuez et al., 2016). Midkine contains HIF-response elements in its promoter and is positively regulated by HIF-1a to favor angiogenesis in embryonic mouse lungs (Reynolds et al., 2004). In addition, endothelial cell proliferation is reduced in hif1a zebrafish mutant hearts (Marin-Juez et al., 2019). These observations indicate that the reduced endothelial proliferation in $m d k a^{c n 105}$ hearts is due to decreased hifla expression. Fast revascularization is crucial for regeneration, since it provides the vascular network necessary for repopulation of the injured area, and coronary vasculature regeneration depends on epicardial signaling (Lavine et al., 2005; Kim et al., 2010). Hence, $m d k a$, a secreted injury-induced cytokine, could act as a paracrine factor released from the epicardium and epicardial-derived fibroblast to induce revascularization of the damaged tissue.

Our analysis reveals that $m d k a$ is readily activated upon injury and is required for zebrafish heart regeneration. Thus, loss of $m d k a$ results in dysregulation of the fibrotic response and retention of the scar as well as defects in angiogenesis, leading to arrest of regeneration. These results suggest that $m d k a$ promotes cardiac remodeling through the regulation of collagen turnover and vascularization and show that regulated attenuation of ECM production is an essential step for fibrosis regression and complete regeneration.

\section{MATERIALS AND METHODS}

\section{Zebrafish Husbandry and Transgenic Lines}

Animal studies were approved by the CNIC Animal Experimentation Ethics Committee and by the Community of Madrid (Ref. PROEX 83.8/20). Animal procedures conformed to EU Directive 2010/63EU and Recommendation 2007/526/EC regarding the protection of animals used for experimental and scientific purposes, enforced in Spanish law under Real Decreto 53/2013. Zebrafish were raised under standard conditions at $28^{\circ} \mathrm{C}$ (Kimmel et al., 1995). Fish lines used were the wild-type $\mathrm{AB}$ strain (ZIRC) and $\mathrm{Tg}(w t 1 b: E G F P)^{l i 1}$ (Perner et al., 2007).

\section{Generation of $\boldsymbol{m d k a}$ Mutant Alleles}

$m d k a-K O$ zebrafish were generated using the oligonucleotides CACCATAGAGC CACTCCGCACAGT and AAACACTGTGC GGAGTGGCTCTAT, targeting the ACTGTGCGGAGTGG CTCTAT sequence. The oligos were inserted into the pX330 vector (Cong et al., 2013), which was linearized with BbsI (New England Biolabs, Ipswich, MA). The guide mRNA was amplified with the forward primer ACGGGGTAATACGACTCACTATAGGGATAGAGCCACTCC GCACAG, which includes T7 polymerase promoter-specific sequences, and the reverse primer AAAAAGCACCGACTCGGT GCCA. The guide RNA was injected into one-cell stage embryos together with Cas9 protein (NEB). Mutants were identified by PCR using the following primers: mdkaFwd TGTTATGTATGATTCTGCGAT and mdka-Rvs ACAGAGGCACAAAACTACCAA. The PCR product was examined in an agarose gel and the mutant animals were identified by the size difference of the PCR products. From the examined injected embryos, 91\% were carrying deletions or insertions.

\section{Heart Cryoinjury and Fin Amputation}

Fish were anaesthetized by immersion in fish water containing $0.04 \%$ tricaine (Sigma-Aldrich, St Louis, MO) and placed on a wet sponge under a dissecting microscope with the ventral side exposed. The cardiac cavity was dissected using microscissors and microforceps, and the pericardium was removed. The ventricle of the heart was exposed and dried and was then touched with a copper probe previously immersed in liquid nitrogen (Gonzalez-Rosa and Mercader, 2012). The fish were immediately returned to water to recover. Amputation of the caudal fin for regeneration experiments was performed as described (Munch et al., 2013). Briefly, fish were anaesthetized in $0.04 \%$ tricaine, and half of the caudal fin was amputated. 


\section{Bromodeoxyuridine Injection}

Adult fish were anaesthetized and placed on a wet sponge under a dissecting microscope. 5-bromo-2'-deoxyuridine (BrdU, SigmaAldrich, B5002) was diluted in phosphate-buffered saline (PBS) to $2.5 \mathrm{mg} / \mathrm{ml}$, and $30 \mu \mathrm{l}$ were injected intraperitoneally $24 \mathrm{~h}$ before heart dissection.

\section{Histology, ISH and Immunohistochemistry}

Acid Fuchsin Orange G (AFOG) staining, in situ hybridization (ISH), fluorescent ISH, and whole-mount in situ hybridization (WM-ISH) were performed as described (Munch et al., 2017; Grivas et al., 2020). Primers for riboprobes used in this study are listed in Supplementary Table 2. For immunofluorescence, sections of paraffin-embedded tissue or cryosections were permeabilized with PBT (PBS containing 0.01\% TritonX-100) and washed with PBS before incubation in blocking solution ( $2 \%$ bovine serum albumin, $10 \%$ goat serum, and $2 \mathrm{mM}$ $\mathrm{MgCl}_{2}$ in $\left.\mathrm{PBS}\right)$. Sections were then incubated overnight at $4^{\circ} \mathrm{C}$ with primary antibodies targeting BrdU (BD Transduction Laboratories, 347580, 1:30), GFP (Aves Labs, Tigard, GFP1010, 1:200), Aldh1a2 (Gene Tex, GTX124302, 1:200), MEF2 (Santa Cruz Biotechnology, Santa Cruz, CA, sc-313, 1:100), phosho-Smad3 (Abcam, Cambridge, MA, ab52903, 1:200), or Mdka, 1:200 (Calinescu et al., 2009). Sections were then incubated with the appropriate secondary antibody and mounted after DAPI staining.

\section{Western Blot (WB)}

Protein expression analysis by WB was performed as described (Grivas et al., 2020), using antibodies to Mdka (1:500) (Calinescu et al., 2009) and alpha-tubulin (Thermo Fisher Scientific, 62204, 1:5000).

\section{Image Analysis and Quantification}

To analyze cardiomyocyte proliferation, $\mathrm{MEF}^{+} / \mathrm{BrdU}^{+}$nuclei were counted in a $100 \mu \mathrm{m}$ area around the injury and normalized to the total $\mathrm{MEF}^{+}$cells. For the analysis of epicardial cell proliferation, epicardial/BrdU ${ }^{+}$cells were counted and normalized to all the epicardial cells. For the coronary endothelial cell proliferation analysis, $\mathrm{GFP}^{+} / \mathrm{BrdU}^{+}$cells were counted within a $200 \mu \mathrm{m}$ radius of the injury site and normalized to the total GFP+ cells. For psmad3 quantification, psmad3+/epicardial cells were measured and normalized to the total epicardial cells. To quantify the scar area, the damaged (fibrotic tissue and collagen) and healthy (myocyte) areas were measured and normalized to the total ventricular area. The collagen-scar area index was calculated as the ratio of the collagen area to the whole scar area. At least three sections from the middle of the ventricle, containing at least on valve as anatomical references, of each heart were used for quantifications. For fin regeneration analysis, we measured the outgrowth of the regenerating fin from the amputation plane to the distal tip of the rays. All analyses were performed using Fiji (ImageJ, NIH).

\section{Gene Expression Analysis}

Gene expression was analyzed by qPCR using the power SYBR Green Master Mix (Applied Biosystems, Foster City, CA) and the ABI PRISM 7900HT Real-Time PCR System. Each condition was analyzed using 3-4 biological replicates with 3 technical replicates per sample. Measurements were normalized to the expression of $18 \mathrm{~s}$ (in embryos) or of elf1a (in adult heart). All primers used for qPCR analysis are listed in Supplementary Table 3. RNA-seq analysis was conducted with 3 pools of 3 apexes from $\mathrm{mdka}^{+/+}$or $m d k a^{c n 105}$ hearts. cDNA libraries were prepared with the NEBNext Ultra II Directional RNA Library Prep Kit (New England Biolabs) and sequenced on a HiSeq 4000 system (Illumina) to generate 60 base single reads, and data were processed with RTA v1.18.66.3. FastQ files for each sample were obtained using bcl2fastq v2.20.0.422 software (Illumina). The resulting reads were mapped against the reference transcriptome GRCz11.99, and gene expression levels were estimated with RSEM (Li and Dewey, 2011). A single pairwise contrast was performed (KO vs. WT). To increase the number of potentially relevant genes, $p$-values were not corrected for multiple testing, and changes in gene expression were considered significant if associated with a raw $P$-value $<0.05$. qPCR provided complementary evidence for the significant differential expression of selected genes, as described above. The resulting collection of 2,029 differentially expressed genes was used for functional enrichment analysis with IPA to derive overrepresented gene lists from Ingenuity's proprietary knowledge-base (IPAKB). IPAKB-derived gene lists consist of collections of genes belonging to the same signaling or metabolic pathway (Canonical Pathway analyses) that either are regulated by the same molecule (Upstream Regulator analyses) or are associated with the same disease or biological function (Downstream Effect analysis). Enrichments associated with a Benjamini-Hochberg adjusted $P$-value $<0.05$ were considered significant. Depending on the number or the type of genes involved, IPA also issued predictions about the activation state of pathways or regulators in the form of a parameter called the z-score; activation or inhibition is indicated by positive or negative values, respectively. Circular plots, summarizing the relationship between selected regulators and target differentially expressed genes were generated with the $\mathrm{R}$ package GOplot (Walter et al., 2015).

\section{Statistical Analysis}

Sample sizes, statistical tests, and $P$-values are specified in the figure legends and were determined with GraphPad Prism software (GraphPad Software Inc., San Diego, CA). Statistical $t$-tests were two-tailed. Differences were considered statistically significant at $P<0.05$.

\section{DATA AVAILABILITY STATEMENT}

Data are deposited in the NCBI GEO database under Accession Number GSE166732. 


\section{ETHICS STATEMENT}

The animal study was reviewed and approved by Animal studies were approved by the CNIC Animal Experimentation Ethics Committee and by the Community of Madrid (Ref. PROEX 83.8/20). Animal procedures conformed to EU Directive 2010/63EU and Recommendation 2007/526/EC regarding the protection of animals used for experimental and scientific purposes, enforced in Spanish law under Real Decreto 53/2013.

\section{AUTHOR CONTRIBUTIONS}

DG and ÁG-R performed experiments. DG and JLP designed the experiments, reviewed all the data, prepared the figures, and wrote the manuscript. All authors reviewed the manuscript during its preparation.

\section{FUNDING}

This study was supported by Grants PID2019-104776RB-I00, CB16/11/00399 (CIBER CV) and RD16/0011/0021 (TERCEL) from the Spanish Ministry of Science, Innovation and Universities (MCIU) and Grants from the Fundación BBVA (Ref.: BIO14_298), Fundación La Marató (Ref.: 20153431) and the European Commission through CardioNeT (Ref.: 28600) from the European Commission to JLP. DG held a Ph.D. fellowship linked to the Grant CardioNeT grant. The cost of this publication was supported in part with funds from the European Regional Development Fund. The CNIC was supported by the Instituto de Salud Carlos III (ISCIII), the MCIU and the Pro CNIC Foundation, and is a Severo Ochoa Centre of Excellence (SEV-2015-0505).

\section{ACKNOWLEDGMENTS}

We thank E. Dìa at the CNIC animal facility for fish husbandry; B. Rios, V. García, and L. Méndez for technical support; the CNIC Microscopy Unit; P. Hitchcock for sharing the Mdka antibody; and S. Bartlett (CNIC) for English editing. RNA-seq was performed by the CNIC Genomics Unit. Data analysis was

\section{REFERENCES}

Ang, N. B., Saera-Vila, A., Walsh, C., Hitchcock, P. F., Kahana, A., Thummel, R., et al. (2020). Midkine-a functions as a universal regulator of proliferation during epimorphic regeneration in adult zebrafish. PLoS One 15:e232308. doi: 10.1371/journal.pone.0232308

Benjamin, E. J., Muntner, P., Alonso, A., Bittencourt, M. S., Callaway, C. W., Carson, A. P., et al. (2019). Heart disease and stroke statistics-2019 update: a report from the american heart association. Circulation 139:e00056-528.

Calinescu, A. A., Raymond, P. A., and Hitchcock, P. F. (2009). Midkine expression is regulated by the circadian clock in the retina of the zebrafish. Vis. Neurosci. 26, 495-501. doi: 10.1017/s0952523809990204

Cao, J., Navis, A., Cox, B. D., Dickson, A. L., Gemberling, M., Karra, R., et al. (2016). Single epicardial cell transcriptome sequencing identifies Caveolin 1 as an essential factor in zebrafish heart regeneration. Development 143, 232-243. doi: 10.1242/dev.130534 carried out by the CNIC Bioinformatics Units, and we especially thank Manuel J. Gómez for his help.

\section{SUPPLEMENTARY MATERIAL}

The Supplementary Material for this article can be found online at: https://www.frontiersin.org/articles/10.3389/fcell.2021. 669439/full\#supplementary-material

Supplementary Figure 1 | Expression of $m d k a$ in regenerating hearts. ISH of mdka in $1 \mathrm{dpci}\left(\mathbf{A}, \mathbf{A}^{\prime}\right), 14 \mathrm{dpci}\left(\mathbf{B}, \mathbf{B}^{\prime}\right)$, and $130 \mathrm{dpci}\left(\mathbf{C}, \mathbf{C}^{\prime}\right)$ hearts. V, ventricle; b.a., bulbus arteriosus. Scale bars, $100 \mu \mathrm{m}$.

Supplementary Figure 2 | Analysis of $m d k b$ in $m d k a^{c n 105}$ embryos and injured hearts. (A,B) WM-ISH of $m d k b$ in $2 \mathrm{dpf} m d k a^{+/+}$or $m d k a^{c n 105}$ embryos. (C,D) ISH of $m d k b$ in 7 dpci $m d k a^{+/+}$or $m d k a^{c n 105}$ hearts. Scale bars (A,B) $200 \mu \mathrm{m}$; (C,D) $100 \mu \mathrm{m}$.

Supplementary Figure $\mathbf{3} \mid m d k^{c n 105}$ phenotype 90 dpci. AFOG staining of 90 dpci $m d k^{+/+}, m k^{+/ c n 105}$, and $m d k a^{c n 105}$ heart sections. Collagen in blue, fibrin in red, and healthy myocardium in brown. Section that contained at least one of the atrioventricular or the bulboventricular valves, were used for quantification of the scar area. Scale bar, $100 \mu \mathrm{m}$.

Supplementary Figure 4 | Monitoring fin regeneration in $m d k a^{c n 105}$ zebrafish. Fin regeneration at $3 \mathrm{dpa}(\mathbf{A}, \mathbf{B}), 7 \mathrm{dpa}(\mathbf{C}, \mathbf{D})$, and $14 \mathrm{dpa}(\mathbf{E}, \mathbf{F})$ in $\mathrm{mdka}^{+/+}$and mdka ${ }^{c n 105}$ fish. Dashed lines indicate the amputation plane. Scale bars, $500 \mu \mathrm{m}$. (G) Quantification of fin outgrowth. $3 \mathrm{dpa}, n_{\mathrm{WT}}=10, n_{\mathrm{KO}}=8$; $7 \mathrm{dpa}, n_{\mathrm{WT}}=5$, $n_{\mathrm{KO}}=6 ; 14 \mathrm{dpa}, n_{\mathrm{WT}}=3, n_{\mathrm{KO}}=5 ;$-test; ${ }^{*} P<0.05$; Mean \pm SD.

Supplementary Figure $\mathbf{5}$ | RNA-seq analysis of 7 dpci hearts. RNA-seq log fold changes of ECM components and angiogenesis genes.

Supplementary Figure 6 | Analysis of scar area and collagen in $7 \mathrm{dpci} m \mathrm{ka}^{+/+}$ and $m d k a^{c n 105}$. (A,B) AFOG staining of $7 \mathrm{dpci} m d \mathrm{ka}^{+/+}$and $m d \mathrm{ka}^{\mathrm{cn} 105}$ hearts. Collagen is shown in blue, fibrin in red, and healthy myocardium in brown. Scale bar, $100 \mu \mathrm{m}$. (C) Quantification of the scar area normalized to the total ventricle area. $n_{W T}=n_{K O}=4$. (D) Percentage of collagen in the scar area. $n_{W T}=4$, $n_{\mathrm{KO}}=5 ;$-test; ${ }^{*} P<0.05 ;$ Mean $\pm S \mathrm{~S}$.

Supplementary Figure 7 | Phosphor-Smad3 quantification, epicardial cell and cardiomyocyte proliferation. (A,B) Immunofluorescence staining of $m \mathrm{dka}^{+/+}$and mdka ${ }^{\text {cn105 }} 7$ dpci Tg(wt1b:GFP) heart sections for phosphor-Smad3 (psmad3, red) and GFP (green). (C) Quantification of psmad3 ${ }^{+}$epicardial cells (Epi). $n_{\mathrm{WT}}=7, n_{\mathrm{KO}}=6$; $t$-test; Mean \pm SD. (D,E) Immunofluorescence staining of $m d k a^{+/+}$and $m d k a^{c n 105} 7 \mathrm{dpci}$ Tg(wt1b:GFP) heart sections for BrdU (red), GFP (green), and Aldh1a2 (white). (F) Quantification of $\mathrm{BrdU}^{+}$epicardial cells. $n_{W T}=10, n_{K O}=11 ; t$-test; Mean \pm SD. (G,H) Immunofluorescence staining of $\mathrm{BrdU}$ and MEF2 in $7 \mathrm{dpci} m \mathrm{mka}^{+/+}$and $m d \mathrm{ka}^{\mathrm{cn} 105}$ hearts. Dashed lines indicate the quantification area. (I) Quantification of $\mathrm{BrdU}^{+} / \mathrm{MEF}^{+}$cardiomyocytes (CM). $n_{\mathrm{WT}}=6, n_{\mathrm{KO}}=7$; $t$-test; Mean \pm SD; Scale bars, $100 \mu \mathrm{m}$.

Chablais, F., and Jazwinska, A. (2012a). Induction of myocardial infarction in adult zebrafish using cryoinjury. J. Vis. Exp. 62:3666.

Chablais, F., and Jazwinska, A. (2012b). The regenerative capacity of the zebrafish heart is dependent on TGFbeta signaling. Development 139, 1921-1930. doi: $10.1242 /$ dev. 078543

Cong, L., Ran, F. A., Cox, D., Lin, S., Barretto, R., Habib, N., et al. (2013). Multiplex genome engineering using CRISPR/Cas systems. Science 339, 819-823.

Dixon, I. M. C., Landry, N. M., and Rattan, S. G. (2019). Periostin reexpression in heart disease contributes to cardiac interstitial remodeling by supporting the cardiac myofibroblast phenotype. Adv. Exp. Med. Biol. 1132, 35-41. doi: 10.1007/978-981-13-6657-4_4

Fukui, S., Kitagawa-Sakakida, S., Kawamata, S., Matsumiya, G., Kawaguchi, N., Matsuura, N., et al. (2008). Therapeutic effect of midkine on cardiac remodeling in infarcted rat hearts. Ann. Thorac. Surg. 85, 562-570. doi: 10.1016/j. athoracsur.2007.06.002 
Gerri, C., Marin-Juez, R., Marass, M., Marks, A., Maischein, H. M., and Stainier, D. Y. R. (2017). Hif-1alpha regulates macrophage-endothelial interactions during blood vessel development in zebrafish. Nat. Commun. $8: 15492$.

Gilkes, D. M., Bajpai, S., Chaturvedi, P., Wirtz, D., and Semenza, G. L. (2013). Hypoxia-inducible factor 1 (HIF-1) promotes extracellular matrix remodeling under hypoxic conditions by inducing P4HA1, P4HA2, and PLOD2 expression in fibroblasts. J. Biol. Chem. 288, 10819-10829. doi: 10.1074/jbc.m112. 442939

Gonzalez-Rosa, J. M., Martin, V., Peralta, M., Torres, M., and Mercader, N. (2011). Extensive scar formation and regression during heart regeneration after cryoinjury in zebrafish. Development 138, 1663-1674. doi: 10.1242/dev.060897

Gonzalez-Rosa, J. M., and Mercader, N. (2012). Cryoinjury as a myocardial infarction model for the study of cardiac regeneration in the zebrafish. Nat. Protoc. 7, 782-788. doi: 10.1038/nprot.2012.025

Grivas, D., Gonzalez-Rajal, A., Guerrero Rodriguez, C., Garcia, R., and de la Pompa, J. L. (2020). Loss of Caveolin-1 and caveolae leads to increased cardiac cell stiffness and functional decline of the adult zebrafish heart. Sci. Rep. 10:12816.

Harrison, M. R., Bussmann, J., Huang, Y., Zhao, L., Osorio, A., Burns, C. G., et al. (2015). Chemokine-guided angiogenesis directs coronary vasculature formation in zebrafish. Dev. Cell 33, 442-454. doi: 10.1016/j.devcel.2015.04.001

Holmes, D. F., Lu, Y., Starborg, T., and Kadler, K. E. (2018). Collagen fibril assembly and function. Curr. Top. Dev. Biol. 130, 107-142. doi: 10.1016/bs.ctdb.2018.02. 004

Horiba, M., Kadomatsu, K., Yasui, K., Lee, J. K., Takenaka, H., Sumida, A., et al. (2006). Midkine plays a protective role against cardiac ischemia/reperfusion injury through a reduction of apoptotic reaction. Circulation 114, 1713-1720. doi: 10.1161/circulationaha.106.632273

Ikutomo, M., Sakakima, H., Matsuda, F., and Yoshida, Y. (2014). Midkine-deficient mice delayed degeneration and regeneration after skeletal muscle injury. Acta Histochem. 116, 319-326. doi: 10.1016/j.acthis.2013.08.009

Ishiguro, H., Horiba, M., Takenaka, H., Sumida, A., Opthof, T., Ishiguro, Y. S., et al. (2011). A single intracoronary injection of midkine reduces ischemia/reperfusion injury in Swine hearts: a novel therapeutic approach for acute coronary syndrome. Front. Physiol. 2:27. doi: 10.3389/fphys.2011.00027

Ito, K., Morioka, M., Kimura, S., Tasaki, M., Inohaya, K., and Kudo, A. (2014). Differential reparative phenotypes between zebrafish and medaka after cardiac injury. Dev. Dyn. 243, 1106-1115. doi: 10.1002/dvdy.24154

Kendall, R. T., and Feghali-Bostwick, C. A. (2014). Fibroblasts in fibrosis: novel roles and mediators. Front. Pharmacol. 5:123. doi: 10.3389/fphar.2014.00123

Kikuchi, K., Holdway, J. E., Major, R. J., Blum, N., Dahn, R. D., Begemann, G., et al. (2011). Retinoic acid production by endocardium and epicardium is an injury response essential for zebrafish heart regeneration. Dev. Cell 20, 397-404. doi: 10.1016/j.devcel.2011.01.010

Kim, J., Wu, Q., Zhang, Y., Wiens, K. M., Huang, Y., Rubin, N., et al. (2010). PDGF signaling is required for epicardial function and blood vessel formation in regenerating zebrafish hearts. Proc. Natl. Acad. Sci. U.S.A. 107, 17206-17210. doi: 10.1073/pnas.0915016107

Kimmel, C. B., Ballard, W. W., Kimmel, S. R., Ullmann, B., and Schilling, T. F. (1995). Stages of embryonic development of the zebrafish. Dev. Dyn. 203, 253-310. doi: 10.1002/aja.1002030302

Lavine, K. J., Yu, K., White, A. C., Zhang, X., Smith, C., Partanen, J., et al. (2005). Endocardial and epicardial derived FGF signals regulate myocardial proliferation and differentiation in vivo. Dev. Cell 8, 85-95. doi: 10.1016/j. devcel.2004.12.002

Li, B., and Dewey, C. N. (2011). RSEM: accurate transcript quantification from RNA-Seq data with or without a reference genome. BMC Bioinformatics 12:323. doi: 10.1186/1471-2105-12-323

Li, J., Wang, Y., Ma, M., Jiang, S., Zhang, X., Zhang, Y., et al. (2019). Autocrine CTHRC1 activates hepatic stellate cells and promotes liver fibrosis by activating TGF-beta signaling. EBioMedicine 40, 43-55. doi: 10.1016/j.ebiom.2019.01.009

Lien, C. L., Schebesta, M., Makino, S., Weber, G. J., and Keating, M. T. (2006). Gene expression analysis of zebrafish heart regeneration. PLoS Biol. 4:e260. doi: 10.1371/journal.pbio.0040260

Luo, J., Uribe, R. A., Hayton, S., Calinescu, A. A., Gross, J. M., and Hitchcock, P. F. (2012). Midkine-A functions upstream of Id2a to regulate cell cycle kinetics in the developing vertebrate retina. Neural Dev. 7:33. doi: 10.1186/1749-81047-33

Marin-Juez, R., El-Sammak, H., Helker, C. S. M., Kamezaki, A., Mullapuli, S. T., Bibli, S. I., et al. (2019). Coronary revascularization during heart regeneration is regulated by epicardial and endocardial cues and forms a scaffold for cardiomyocyte repopulation. Dev. Cell 51:e504.

Marin-Juez, R., Marass, M., Gauvrit, S., Rossi, A., Lai, S. L., Materna, S. C., et al. (2016). Fast revascularization of the injured area is essential to support zebrafish heart regeneration. Proc. Natl. Acad. Sci. U.S.A. 113, 11237-11242. doi: $10.1073 /$ pnas. 1605431113

Marro, J., Pfefferli, C., de Preux Charles, A. S., Bise, T., and Jazwinska, A. (2016). Collagen XII contributes to epicardial and connective tissues in the Zebrafish heart during ontogenesis and regeneration. PLoS One 11:e0165497. doi: 10. 1371/journal.pone.0165497

Moustakas, A., and Heldin, C. H. (2009). The regulation of TGFbeta signal transduction. Development 136, 3699-3714.

Munch, J., Gonzalez-Rajal, A., and de la Pompa, J. L. (2013). Notch regulates blastema proliferation and prevents differentiation during adult zebrafish fin regeneration. Development 140, 1402-1411. doi: 10.1242/dev.087346

Munch, J., Grivas, D., Gonzalez-Rajal, A., Torregrosa-Carrion, R., and de la Pompa, J. L. (2017). Notch signalling restricts inflammation and serpinel expression in the dynamic endocardium of the regenerating zebrafish heart. Development 144, 1425-1440. doi: 10.1242/dev.143362

Nagashima, M., D’Cruz, T. S., Danku, A. E., Hesse, D., Sifuentes, C., Raymond, P. A., et al. (2020). Midkine-a is required for cell cycle progression of muller glia during neuronal regeneration in the vertebrate retina. J. Neurosci. 40, 1232-1247. doi: 10.1523/jneurosci.1675-19.2019

Obama, H., Biro, S., Tashiro, T., Tsutsui, J., Ozawa, M., Yoshida, H., et al. (1998). Myocardial infarction induces expression of midkine, a heparin-binding growth factor with reparative activity. Anticancer Res. 18, 145-152.

Perner, B., Englert, C., and Bollig, F. (2007). The Wilms tumor genes wt1a and wt1b control different steps during formation of the zebrafish pronephros. Dev. Biol. 309, 87-96. doi: 10.1016/j.ydbio.2007.06.022

Poss, K. D., Wilson, L. G., and Keating, M. T. (2002). Heart regeneration in zebrafish. Science 298, 2188-2190. doi: 10.1126/science.1077857

Reynolds, P. R., Mucenski, M. L., Le Cras, T. D., Nichols, W. C., and Whitsett, J. A. (2004). Midkine is regulated by hypoxia and causes pulmonary vascular remodeling. J. Biol. Chem. 279, 37124-37132. doi: 10.1074/jbc.m4052 54200

Sanchez-Iranzo, H., Galardi-Castilla, M., Sanz-Morejon, A., Gonzalez-Rosa, J. M., Costa, R., Ernst, A., et al. (2018). Transient fibrosis resolves via fibroblast inactivation in the regenerating zebrafish heart. Proc. Natl. Acad. Sci. U.S.A. 115, 4188-4193. doi: 10.1073/pnas.1716713115

Sanz-Morejon, A., Garcia-Redondo, A. B., Reuter, H., Marques, I. J., Bates, T., Galardi-Castilla, M., et al. (2019). Wilms tumor 1b expression defines a proregenerative macrophage subtype and is required for organ regeneration in the zebrafish. Cell Rep. 28, 1296.e6-1306.e6.

Schnabel, K., Wu, C. C., Kurth, T., and Weidinger, G. (2011). Regeneration of cryoinjury induced necrotic heart lesions in zebrafish is associated with epicardial activation and cardiomyocyte proliferation. PLoS One 6:e18503. doi: 10.1371/journal.pone.0018503

Shimazaki, M., Nakamura, K., Kii, I., Kashima, T., Amizuka, N., Li, M., et al. (2008). Periostin is essential for cardiac healing after acute myocardial infarction. J. Exp. Med. 205, 295-303.

Sorci-Thomas, M. G., Pollard, R. D., and Thomas, M. J. (2015). What does procollagen $\mathrm{C}$-endopeptidase enhancer protein 2 have to do with HDLcholesteryl ester uptake? Or how I learned to stop worrying and love reverse cholesterol transport? Curr. Opin. Lipidol. 26, 420-425. doi: 10.1097/mol. 0000000000000211

Sumi, Y., Muramatsu, H., Takei, Y., Hata, K., Ueda, M., and Muramatsu, T. (2002). Midkine, a heparin-binding growth factor, promotes growth and glycosaminoglycan synthesis of endothelial cells through its action on smooth muscle cells in an artificial blood vessel model. J. Cell Sci. 115, 2659-2667.

Sumida, A., Horiba, M., Ishiguro, H., Takenaka, H., Ueda, N., Ooboshi, H., et al. (2010). Midkine gene transfer after myocardial infarction in rats prevents remodelling and ameliorates cardiac dysfunction. Cardiovasc. Res. 86, 113-121. doi: $10.1093 / \mathrm{cvr} / \mathrm{cvp} 386$ 
Takenaka, H., Horiba, M., Ishiguro, H., Sumida, A., Hojo, M., Usui, A., et al. (2009). Midkine prevents ventricular remodeling and improves long-term survival after myocardial infarction. Am. J. Physiol. Heart Circ. Physiol. 296, H462-H469.

Theocharis, A. D., Skandalis, S. S., Gialeli, C., and Karamanos, N. K. (2016). Extracellular matrix structure. Adv. Drug Deliv. Rev. 97, 4-27.

Verrecchia, F., Vindevoghel, L., Lechleider, R. J., Uitto, J., Roberts, A. B., and Mauviel, A. (2001). Smad3/AP-1 interactions control transcriptional responses to TGF-beta in a promoter-specific manner. Oncogene 20, 3332-3340. doi: 10.1038/sj.onc. 1204448

Walter, W., Sanchez-Cabo, F., and Ricote, M. (2015). GOplot: an R package for visually combining expression data with functional analysis. Bioinformatics 31 , 2912-2914. doi: 10.1093/bioinformatics/btv300

Wang, J., Karra, R., Dickson, A. L., and Poss, K. D. (2013). Fibronectin is deposited by injury-activated epicardial cells and is necessary for zebrafish heart regeneration. Dev. Biol. 382, 427-435. doi: 10.1016/j.ydbio.2013.08.012

Winkler, C., Schafer, M., Duschl, J., Schartl, M., and Volff, J. N. (2003). Functional divergence of two zebrafish midkine growth factors following fish-specific gene duplication. Genome Res. 13, 1067-1081. doi: 10.1101/gr.1097503
Xu, S., Webb, S. E., Lau, T. C. K., and Cheng, S. H. (2018). Matrix metalloproteinases (MMPs) mediate leukocyte recruitment during the inflammatory phase of zebrafish heart regeneration. Sci. Rep. 8:7199.

Yamada, H., Inazumi, T., Tajima, S., Muramatsu, H., and Muramatsu, T. (1997). Stimulation of collagen expression and glycosaminoglycan synthesis by midkine in human skin fibroblasts. Arch. Dermatol. Res. 289, 429-433. doi: $10.1007 / \mathrm{s} 004030050216$

Conflict of Interest: The authors declare that the research was conducted in the absence of any commercial or financial relationships that could be construed as a potential conflict of interest.

Copyright (c) 2021 Grivas, González-Rajal and de la Pompa. This is an open-access article distributed under the terms of the Creative Commons Attribution License (CC BY). The use, distribution or reproduction in other forums is permitted, provided the original author(s) and the copyright owner(s) are credited and that the original publication in this journal is cited, in accordance with accepted academic practice. No use, distribution or reproduction is permitted which does not comply with these terms. 